\title{
On the Internal Resonance Modes of Time Domain Surface Integral Equations for Acoustic Transmission Problems
}

\author{
Rui Chen* and Hakan Bagci \\ Division of Computer, Electrical, and Mathematical Science and Engineering, \\ King Abdullah University of Science and Technology (KAUST), \\ Thuwal 23955-6900, Saudi Arabia \\ \{rui.chen, hakan.bagci\}@kaust.edu.sa
}

\begin{abstract}
The internal resonance problem pertinent to time domain surface integral equations (SIEs) for analyzing transient acoustic scattering from penetrable objects is investigated. The first equation considered here is constructed by combining SIE representations of the internal and external problems via the continuity of the velocity potential and its normal derivative. Just like its frequency-domain counterpart, this equation suffers from the internal resonance problem. But it is demonstrated in this work that, unlike the frequency-domain solution, by increasing the accuracy of the discretization, the amplitude of these spurious modes can be suppressed to a level that does not significantly affect the solution. The second equation considered here is obtained by linearly combining the first equation with its normal derivative and its solution is completely free from spurious internal resonance modes.
\end{abstract}

\section{Introduction}

The internal resonance problem pertinent to frequencydomain surface integral equations (SIEs) for analyzing time-harmonic acoustic scattering has been extensively studied [?, ?, ?]. The internal (spurious) resonance modes are observed in the solution when the excitation frequency overlaps with the resonance frequencies of the acoustically soft cavity that has the same shape as the scatterer [?]. On the other hand, ideally, the solution of the time domain surface integral equations (TDSIEs) (for analyzing transient acoustic scattering) should be free from these resonance modes since these purely oscillating modes do not satisfy the zero-initial conditions of time marching schemes [?]. But yet, time domain solutions are corrupted by them. It has been conjectured that this is due to the built-up numerical errors resulting from the discretization [?].

In this work, this conjecture is investigated for acoustic transmission problems. The first TDSIE considered here is constructed by combining SIE representations of the internal and external problems (interior and exterior of the acoustically penetrable scatterer) via the continuity of the velocity potential and its normal derivative. Here, this equation is termed the time domain "regular" potential integral equation (TDRPIE). Its well-known that TDRPIE's frequency-domain counterpart suffers from the internal resonance problem [?]. However, in this work, the conjecture mentioned above is verified. More specifically, it is demonstrated that, by increasing the accuracy of the discretization, the amplitude of internal resonance modes in the time domain could be suppressed to a level that does not significantly affect the solution. This increase in the accuracy is obtained using approximately band- and time-limited basis functions in time and a higher-order Nyström discretization in space.

The second equation investigated here is obtained by linearly combining the first equation with its normal derivative. This equation is termed the time domain "combined" potential integral equation (TDCPIE) here. The same scheme is used to discretize it and the numerical results demonstrate that its solution is completely free from spurious internal resonance modes just like its frequencydomain counterpart [?].

\section{Formulation}

Let $S$ denote the surface of a homogeneous penetrable object residing in an unbounded homogeneous background medium. The wave speed and medium density in the background medium and the scatterer are $\left\{c_{1}, \rho_{1}\right\}$ and $\left\{c_{2}, \rho_{2}\right\}$, respectively. An acoustic wave with velocity potential $\varphi^{\mathrm{i}}(\mathbf{r}, t)$ is incident on the object. It is assumed that $\varphi^{\mathrm{i}}(\mathbf{r}, t)$ is band-limited and vanishingly small for $t \leq 0, \forall \mathbf{r} \in S$. The velocity potential on the scatterer surface $\varphi_{1}(\mathbf{r}, t)$ and its normal derivative $\partial_{n} \varphi_{1}(\mathbf{r}, t)$ are related to $\varphi^{\mathrm{i}}(\mathbf{r}, t)$ and $\partial_{n} \varphi^{\mathrm{i}}(\mathbf{r}, t)$ through the TDSIE system as

$$
\begin{aligned}
& C_{11}\left[\varphi_{1}\right](\mathbf{r}, t)+C_{12}\left[\partial_{n} \varphi_{1}\right](\mathbf{r}, t)=b_{1}(\mathbf{r}, t), \mathbf{r} \in S \\
& C_{21}\left[\varphi_{1}\right](\mathbf{r}, t)+C_{22}\left[\partial_{n} \varphi_{1}\right](\mathbf{r}, t)=b_{2}(\mathbf{r}, t), \mathbf{r} \in S,
\end{aligned}
$$

where $\left[\begin{array}{ll}b_{1}(\mathbf{r}, t) & b_{2}(\mathbf{r}, t)\end{array}\right]^{T}=\left[\begin{array}{ll}\varphi^{\mathrm{i}}(\mathbf{r}, t) & 0\end{array}\right]^{T}$ and $\left[\begin{array}{ll}b_{1}(\mathbf{r}, t) & b_{2}(\mathbf{r}, t)\end{array}\right]^{T}=\left[\begin{array}{ll}\alpha_{1} \varphi^{\mathrm{i}}(\mathbf{r}, t) & \beta_{1} \partial_{n} \varphi^{\mathrm{i}}(\mathbf{r}, t)\end{array}\right]^{T}$ for the TDRPIE and TDCPIE, respectively, and the operators $C_{11}[\sigma](\mathbf{r}, t), C_{12}[\sigma](\mathbf{r}, t), C_{21}[\sigma](\mathbf{r}, t)$, and $C_{22}[\sigma](\mathbf{r}, t)$ are 
expressed as

$$
\begin{aligned}
& C_{11}[\sigma](\mathbf{r}, t)=\left\{\begin{array}{l}
\frac{1}{2} \sigma(\mathbf{r}, t)-D_{1}[\sigma](\mathbf{r}, t), \mathrm{TDRPIE} \\
\bar{\alpha} \sigma(\mathbf{r}, t)-\alpha_{1} D_{1}[\sigma]+\alpha_{2} D_{2}[\sigma], \mathrm{TDCPIE}
\end{array}\right. \\
& C_{12}[\sigma](\mathbf{r}, t)=\left\{\begin{array}{l}
S_{1}[\sigma](\mathbf{r}, t), \mathrm{TDRPIE} \\
\alpha_{1} S_{1}[\sigma](\mathbf{r}, t)-\frac{\alpha_{2}}{\chi} S_{2}[\sigma](\mathbf{r}, t), \mathrm{TDCPIE}
\end{array}\right. \\
& C_{21}[\sigma](\mathbf{r}, t)=\left\{\begin{array}{l}
\frac{1}{2} \sigma(\mathbf{r}, t)+D_{2}[\sigma](\mathbf{r}, t), \mathrm{TDRPIE} \\
-\beta_{1} N_{1}[\sigma](\mathbf{r}, t)+\beta_{2} \chi N_{2}[\sigma](\mathbf{r}, t), \mathrm{TDCPIE}
\end{array}\right. \\
& C_{22}[\sigma](\mathbf{r}, t)=\left\{\begin{array}{l}
-\frac{1}{\chi} S_{2}[\sigma](\mathbf{r}, t), \mathrm{TDRPIE} \\
\bar{\beta} \sigma(\mathbf{r}, t)+\beta_{1} D^{\prime}{ }_{1}[\sigma]-\beta_{2} D^{\prime}{ }_{2}[\sigma], \mathrm{TDCPIE}
\end{array}\right.
\end{aligned}
$$

Here, $k \in\{1,2\}, \chi=\rho_{1} / \rho_{2}, \bar{\alpha}=\left(\alpha_{1}+\alpha_{2}\right) / 2, \bar{\beta}=$ $\left(\beta_{1}+\beta_{2}\right) / 2, \alpha_{k}$ and $\beta_{k}$ are constants, and the integral operators $S_{k}[\sigma](\mathbf{r}, t), D_{k}[\sigma](\mathbf{r}, t), D_{k}^{\prime}[\sigma](\mathbf{r}, t)$, and $N_{k}[\sigma](\mathbf{r}, t)$ are expressed as

$$
\begin{aligned}
& S_{k}[\sigma](\mathbf{r}, t)=\int_{S} G_{k}\left(\mathbf{r}, \mathbf{r}^{\prime}, t\right) * \sigma\left(\mathbf{r}^{\prime}, t\right) d \mathbf{r}^{\prime} \\
& D_{k}[\sigma](\mathbf{r}, t)=\text { p.v. } \int_{S} \partial_{n^{\prime}} G_{k}\left(\mathbf{r}, \mathbf{r}^{\prime}, t\right) * \sigma\left(\mathbf{r}^{\prime}, t\right) d \mathbf{r}^{\prime} \\
& D_{k}^{\prime}[\sigma](\mathbf{r}, t)=\text { p.v. } \int_{S} \partial_{n} G_{k}\left(\mathbf{r}, \mathbf{r}^{\prime}, t\right) * \sigma\left(\mathbf{r}^{\prime}, t\right) d \mathbf{r}^{\prime} \\
& N_{k}[\sigma](\mathbf{r}, t)=\int_{S} \partial_{n n^{\prime}}^{2} G_{k}\left(\mathbf{r}, \mathbf{r}^{\prime}, t\right) * \sigma\left(\mathbf{r}^{\prime}, t\right) d \mathbf{r}^{\prime}
\end{aligned}
$$

where $G_{k}\left(\mathbf{r}, \mathbf{r}^{\prime}, t\right)=\delta\left(t-R / c_{k}\right) /(4 \pi R)$ is the time domain Green function, $R=\left|\mathbf{r}-\mathbf{r}^{\prime}\right|$ is the distance between "observer" point $\mathbf{r}$ and "source" point $\mathbf{r}^{\prime}, \partial_{n}=\hat{\mathbf{n}}(\mathbf{r}) \cdot \nabla$ and $\partial_{n^{\prime}}=\hat{\mathbf{n}}^{\prime}\left(\mathbf{r}^{\prime}\right) \cdot \nabla^{\prime}$ are the normal derivatives, and $\hat{\mathbf{n}}(\mathbf{r})$ and $\hat{\mathbf{n}}^{\prime}\left(\mathbf{r}^{\prime}\right)$ are outward pointing unit normal vectors.

To numerically solve (1), $\varphi_{1}(\mathbf{r}, t)$ and $\partial_{n} \varphi_{1}(\mathbf{r}, t)$ are expanded as $[?, ?, ?, ?, ?, ?, ?]$

$$
\begin{array}{r}
\varphi_{1}(\mathbf{r}, t)=\left.\sum_{i=1}^{N_{\mathrm{t}}} \sum_{q=1}^{N_{\mathrm{p}}} \sum_{n=1}^{N_{\mathrm{n}}} \mathbf{I}_{i}^{1}\right|_{q n} \vartheta(\mathbf{r}) \ell_{q n}(\mathbf{r}) T_{i}(t), \\
\partial_{n} \varphi_{1}(\mathbf{r}, t)=\left.\sum_{i=1}^{N_{\mathrm{t}}} \sum_{q=1}^{N_{\mathrm{p}}} \sum_{n=1}^{N_{\mathrm{n}}} \mathbf{I}_{i}^{2}\right|_{q n} \vartheta(\mathbf{r}) \ell_{q n}(\mathbf{r}) T_{i}(t) .
\end{array}
$$

Here, $N_{\mathrm{t}}$ is the number of time steps, $N_{\mathrm{p}}$ is the number of curvilinear triangles used for discretizing $S, N_{\mathrm{n}}$ is the number of interpolation points on each triangle, $\ell_{q n}(\mathbf{r})$ is the Lagrange interpolation function defined at $\mathbf{r}_{q n}$ ( $n^{\text {th }}$ interpolation point on $q^{\text {th }}$ patch) [?], $\vartheta(\mathbf{r})$ is the inverse of Jacobian, $T_{i}(t)=T(t-i \Delta t)$, where $T(t)$ is the temporal basis function constructed using the band-limited approximate prolate spherical wave (APSW) function $[?, ?, ?], \Delta t$ is the time step size, and $\left.\mathbf{I}_{i}^{1}\right|_{q n}$ and $\left.\mathbf{I}_{i}^{2}\right|_{q n}$ are unknown expansion coefficients to be solved for.

Substituting (2) into the temporal derivative form of (1) and point-testing and sampling the resulting equation in space (at $\mathbf{r}_{p m}, p=1, \ldots, N_{\mathrm{p}}, m=1, \ldots, N_{\mathrm{n}}$ ) and time (at $t=j \Delta t$ ), respectively, yield

$$
\mathbf{Z}_{0} \mathbf{I}_{j}=\mathbf{V}_{j}-\sum_{i=1}^{j-1} \mathbf{Z}_{j-i} \mathbf{I}_{i}-\sum_{i=j+1}^{j+N_{\mathrm{hw}}} \mathbf{Z}_{j-i} \mathbf{I}_{i}, j=1, \ldots, N_{\mathrm{t}}
$$

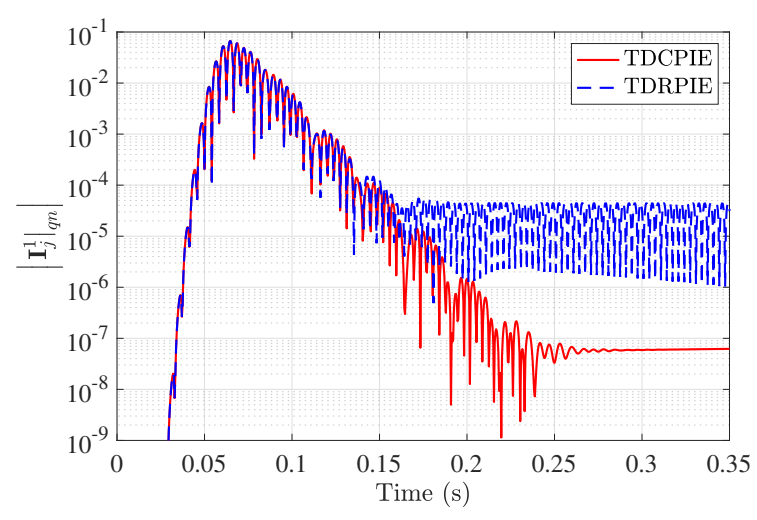

Figure 1. $\left|\mathbf{I}_{j}^{1}\right|_{q n} \mid, q=3, n=3$, calculated using the TDRPIE and TDCPIE at $\mathbf{r}_{q n}=(0.78,0.58,0.23) \mathrm{m}$.

Here, $N_{\mathrm{hw}}$ is the half-width of $T(t), \quad \mathbf{I}_{j}$ and $\mathbf{I}_{i}$ store $\left.\mathbf{I}_{j}^{\mathrm{b}}\right|_{q n}$ and $\left.\mathbf{I}_{i}^{\mathrm{b}}\right|_{q n}$, respectively, $\mathbf{V}_{j}$ stores $\left.\partial_{t} b_{1}\left(\mathbf{r}_{p m}, t\right)\right|_{t=j \Delta t}$ and $\left.\partial_{t} b_{2}\left(\mathbf{r}_{p m}, t\right)\right|_{t=j \Delta t}$, and the entries of $\mathbf{Z}_{j-i}$ 's blocks are given by $\left\{\mathbf{Z}_{j-i}\right\}_{p m, q n}^{\mathrm{ab}}=\left.\partial_{t} C_{\mathrm{ab}}\left[\vartheta \ell_{q n} T_{i}\right]\left(\mathbf{r}_{p m}, t\right)\right|_{t=j \Delta t}$, a, b $\in$ $\{1,2\}$.

Note that (3) is a non-causal system due to the "future" contributions on the right-hand side of (3), i.e., matrix-vector multiplication contributions where $j-i<0$. These contributions stem from the fact $T(t)$ is a "two-sided" interpolation function. This non-causal system is converted into a causal one using an extrapolation scheme $[?, ?, ?]$ for recursively calculating $\mathbf{I}_{j}, j=1, \ldots, N_{\mathrm{t}}$ via time marching.

\section{Numerical Results}

In this example, the scatterer is a unit penetrable sphere centered at the origin. Medium parameters are $\chi=1.5$, $c_{1}=300 \mathrm{~m} / \mathrm{s}$, and $c_{2}=200 \mathrm{~m} / \mathrm{s}$. The incident velocity potential is a Gaussian pulse $\varphi^{\mathrm{i}}(\mathbf{r}, t)=G\left(t-z / c_{1}\right)$, where $G(t)=\cos \left[2 \pi f_{0}\left(t-t_{\mathrm{p}}\right)\right] \exp \left[-\left(t-t_{\mathrm{p}}\right)^{2} /\left(2 \sigma^{2}\right)\right], \sigma=$ $3 /\left(2 \pi f_{\mathrm{bw}}\right)$, and $t_{\mathrm{p}}=10 \sigma$. Here, $f_{0}$ is the center frequency and $f_{\mathrm{bw}}$ is the effective bandwidth. The time step size is chosen as $\Delta t=1 /\left(2 \gamma f_{\max }\right)$ with oversampling factor $\gamma$ and maximum frequency $f_{\max }=f_{0}+f_{\mathrm{bw}}$. The sphere is discretized into $N_{\mathrm{p}}=396$ curvilinear triangles, the order of $\ell_{q n}(\mathbf{r})$ is two $\left(N_{\mathrm{n}}=6\right)$, and $N_{\mathrm{hw}}=7$. The coupling constants are $\alpha_{1}=\alpha_{2}=\beta_{1}=1.0$ and $\beta_{2}=2 / 3$.

For the first simulation, $f_{0}=120 \mathrm{~Hz}, f_{\mathrm{bw}}=80 \mathrm{~Hz}$, and $\gamma=6$ resulting in $\Delta t=0.42 \mathrm{~ms}$. Note that the lowest interior resonance frequency at $150 \mathrm{~Hz}$ is within the band of the excitation [?]. Figure 1 compares $\left|\mathbf{I}_{j}^{1}\right|_{q n} \mid, q=3$, $n=3$, computed using the TDRPIE and TDCPIE at $\mathbf{r}_{q n}=$ $(0.78,0.58,0.23) \mathrm{m}$. It is clearly shown that the solution of the TDRPIE is corrupted by (non-decaying) oscillations (spurious internal resonance modes) while that of the TDCPIE is free of any resonances. 


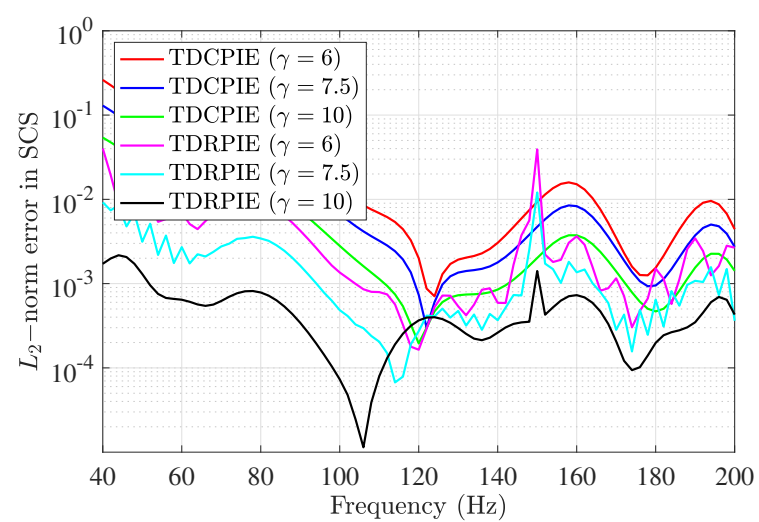

Figure 2. $L_{2}$-norm error in SCS obtained from solutions of the TDRPIE and TDCPIE.

For the second set of simulations, $f_{0}=120 \mathrm{~Hz}, f_{\mathrm{bw}}=$ $80 \mathrm{~Hz}$, and three sets of oversampling factor are considered as $\gamma \in\{6,7.5,10\}$ resulting in $\Delta t=\{0.42,0.33,0.25\} \mathrm{ms}$. Figure 2 compares the $L_{2}$-norm error in the scattering cross section (SCS) obtained from the solutions of the TDRPIE and the TDCPIE at $\theta=\left[0^{\circ}, 180^{\circ}\right]$ and $\phi=0^{\circ}$. Note that the error is computed using the Mie series solution as the reference [?]. As shown in Fig. 2, the solution of the TDRPIE has a spurious resonances at $150 \mathrm{~Hz}$. Additionally, the figure clearly shows that increasing the sampling rate (i.e., using larger $\gamma$ ) could suppress the amplitude of the spurious resonance mode to a level that does not significantly affect the solution of the TDRPIE. On the other hand, Fig. 2 shows that the TDCPIE does not support any resonance modes for any of the sampling rates.

\section{Conclusion}

The internal resonance problem of two types of TDSIEs for acoustically penetrable scatterers is investigated in this work. Numerical results demonstrate that the solution of the TDRPIE is replete with the spurious resonance modes but, unlike its frequency-domain counterpart, the amplitude of these modes can be suppressed with the increase of discretization accuracy. On the other hand, the TDCPIE completely eliminates the internal resonance problem.

\section{Acknowledgements}

This publication is based upon work supported by the King Abdullah University of Science and Technology (KAUST) Office of Sponsored Research (OSR) under Award No 2016-CRG5-2953. The authors would like to thank the King Abdullah University of Science and Technology Supercomputing Laboratory (KSL) for providing the required computational resources.

\section{References}

[1] A. J. Burton and G. F. Miller, "The Application of Integral Equation Methods to the Numerical Solution of
Some Exterior Boundary-value Problems," Proc. R. Soc. London Ser. A, 323, 1553, Jun. 1971, pp. 201210.

[2] H. A. Schenck, "Improved Integral Formulation for Acoustic Radiation Problems," J. Acoust. Soc. Am., 44, 1, Jul. 1968, pp. 41-58.

[3] C.-J. Zheng, H.-B. Chen, H.-F. Gao, and L. Du, "Is the Burton-Miller Formulation Really Free of Fictitious Eigenfrequencies?," Eng. Anal. Bound Elem., 59, Oct. 2015, pp. 43-51.

[4] Y. Shi, H. Bagci, and M. Lu, "On the Internal Resonant Modes in Marching-on-in-time Solution of the Time Domain Electric Field Integral Equation," IEEE Trans. Antennas Propag., 61, 8, Aug. 2013, pp. 43894392.

[5] R. E. Kleinman and P. A. Martin, "On Single Integral Equations for the Transmission Problem of Acoustics," SIAM J. Appl. Math., 48, 2, Apr. 1988, pp. 307325.

[6] G. Kang, J. Song, W. C. Chew, K. C. Donepudi, and J.-M. Jin, "A Novel Grid-robust Higher Order Vector Basis Function for the Method of Moments," IEEE Trans. Antennas Propag., 49, 6, Jun. 2001, pp. 908915.

[7] R. Chen, S. B. Sayed, H. A. Ulku and H. Bagci, "An Explicit Time Marching Scheme for Efficient Solution of the Magnetic Field Integral Equation at Low Frequencies," IEEE Trans. Antennas Propag., 69, 2, Feb. 2021, pp. 1213-1218, doi: 10.1109/TAP.2020.3010997.

[8] R. Chen, S. B. Sayed, N. Alharthi, D. Keyes, and H. Bagci, "An Explicit Marching-on-in-time Scheme for Solving the Time Domain Kirchhoff Integral Equation," J. Acoust. Soc. Am., 146, 3, Sep. 2019, pp. 2068-2079, doi: 10.1121/1.5125259.

[9] R. Chen and H. Bagci, "An Explicit MOT Scheme for Solving the Nyström-discretized TD-MFIE," in Proc. IEEE Int. Symp. Antennas Propag. Soc., Boston, MA, USA, Jul. 2018, pp. 2443-2444, doi: 10.1109/APUSNCURSINRSM.2018.8609242.

[10] R. Chen, S. B. Sayed, and H. Bagci, "An Explicit Marching-on-in-time Scheme for Solving the Kirchhoff Integral Equation," in Proc. IEEE Int. Symp. Antennas Propag. Soc., Boston, MA, USA, Jul. 2018, pp. 2409-2410, doi: 10.1109/APUSNCURSINRSM.2018.8608706.

[11] R. Chen and H. Bagci, "On Higher-order Nyström Discretization of Scalar Potential Integral Equation for Penetrable Scatterers," in Proc. Appl. Comput. Electrom. Symp., Miami, FL, USA, Apr. 2019, pp. 12. 
[12] R. Chen and H. Bagci, "A Higher-order Explicit Marching-on-in-time for Analysis of Transient Acoustic Scattering from Rigid Objects," in Proc. IEEE Int. Symp. Antennas Propag. Soc., Atlanta, GA, USA, Jul. 2019, pp. 179-180, doi: 10.1109/APUSNCURSINRSM.2019.8888657.

[13] S. B. Sayed, H. A. Ulku, and H. Bagci, "A Stable Marching On-in-time Scheme for Solving the Time-domain Electric Field Volume Integral Equation on High-contrast Scatterers," IEEE Trans. Antennas Propag., 63, 7, May 2015, pp. 3098-3110.

[14] D. S. Weile, G. Pisharody, N.-W. Chen, B. Shanker, and E. Michielssen, "A Novel Scheme for the Solution of the Time-domain Integral Equations of Electromagnetics," IEEE Trans. Antennas Propag., 52, 1, Jan. 2004, pp. 283-295.

[15] R. A. Wildman, G. Pisharody, D. S. Weile, S. Balasubramaniam, and E. Michielssen, "An Accurate Scheme for the Solution of the Time-domain Integral Equations of Electromagnetics using Higher Order Vector Bases and Bandlimited Extrapolation," IEEE Trans. Antennas Propag., 52, 11, Nov. 2004, pp. 2973-2984.

[16] S. Turley, "Acoustic Scattering from a Sphere," Class Notes, Department of Physics and Astronomy, Brigham Young University, Provo, UT, 2006. 